\title{
TUBERCULOUS BRONCHO-OESOPHAGEAL FISTULA IN A CHILD
}

\author{
BY \\ E. A. DANinO, C. J. EVANS, and J. H. THOMAS \\ From Morriston Hospital, Swansea
}

(RECEIVED FOR PUBLICATION JULY 16, 1955)

The development of a fistula between the oesophagus and a stem bronchus or one of its subdivisions is rare when malignant disease is excluded. Of the 670 cases of broncho-oesophageal fistula collected from the literature by Monserrat (1941) only 41 were due to infection and 40 to trauma, while 367 were due to neoplasm and 222 to congenital defect. Coleman and Bunch (1950) suggest the following classification:

A. Congenital.

B. Acquired.

1. Malignant.

2. Non-malignant.
a. Trauma.
b. From diverticulum.
c. Infection.

They analysed 75 cases of acquired non-malignant fistulae from the literature, and state that 43 were due to perforation of an oesophageal diverticulum and 14 to tuberculosis. They added four cases of their own, one being a woman of 65 years in whom erosion of a calcified lymph gland caused a fistula between the oesophagus and the posterior segmental bronchus of the left lower lobe. Maier (1951) suggests a similar pathogenesis in a man of 36 , where the presenting feature was haemoptysis: the fistula opened into the right stem bronchus. Nuboer (1953) presents an almost identical case of a man aged 40 , and states that a small minority of acquired non-malignant bronchooesophageal fistulae occur in children. They are usually due to inhalation or ingestion of foreign bodies or corrosives. Morton, Osborne, and Klassen (1950) describe a man aged 26 with symptoms of bronchiectasis since the age of 4 , in whom a fistula opening into the right stem bronchus was discovered at operation. As the lining contained epithelium and the wall smooth muscle, this case was classified as being of the congenital variety, but we feel that it could have been acquired from perforation of an oesophageal diverticulum. The symptoms in this case began in childhood, but we have been unable to find a case in the literature where a definite diagnosis was made under 18 years of age, and in no instance have we found such a lesion associated with a non-calcified enlarged lymph gland. We feel that the following case is worthy of publication in view of its rarity.

\section{CASE REPORT}

A boy, aged 7, was seen at the chest clinic, Swansea, in May, 1948, complaining of a dry cough since an attack of whooping-cough one year before. Radiographs showed atelectasis of the right lower lobe with enlargement of the right hilar and right paratrachea! lymph nodes without evidence of calcification. There was no lesion visible in the remainder of the lung fields. A Mantoux test, using 1/1,000 old tuberculin, was positive, and a diagnosis of primary complex was made.

He remained symptom-free until July, 1952, when he began to expectorate approximately $\frac{1}{2} \mathrm{oz}$. of purulent sputum daily. There was no change in the radiological appearances.

His general condition was seen to be good, although he appeared small for his age. There was no evidence of clubbing of the fingers.

On auscultation numerous coarse crepitations and rhonchi were audible at the right base on deep inspiration and on coughing. Swallowing liquids did not precipitate paroxysms of coughing or increase the amount of sputum and produced no alteration in the physical signs (negative Ono's sign).

Two months later he had a febrile illness with consolidation in the right middle lobe. This resolved satisfactorily with penicillin.

He continued to expectorate a variable quantity of sputum, and an intensive search failed to find tubercle bacilli on either microscopy or culture.

In August, 1953, he was admitted to the Thoracic Surgical Unit at Morriston Hospital, where a routine barium swallow given during fluoroscopic examination of the chest showed that barium entered the collapsed right lower lobe. Lipiodol was then substituted for the barium and radiographs taken. These demonstrated a fistula between the oesophagus and the posterior basal segmental bronchus of the right lower lobe. The only abnormality detected on bronchoscopy was reddening of the mucosa of the right lower lobe 
bronchus with pus exuding from all its segmental orifices. Bronchography showed saccular bronchiectasis of all segments of the right lower lobe. The remainder of the bronchial tree appeared normal. On oesophagoscopy the orifice of the fistula was identified $21 \mathrm{~cm}$. from the upper teeth.

On October 21 thoracotomy was performed after preliminary bronchoscopy and bronchial aspiration. The lower lobe was found to be atelectatic and adherent on all surfaces by dense fibrous adhesions. During subsequent dissection the fistula was identified as a tubular fibrous structure about $1 \mathrm{~cm}$. long and $1 \mathrm{~cm}$. diameter between the oesophagus and the bronchus at a point below the apical bronchus to the lower lobe. Identification of the branches of the pulmonary artery to the lower lobe was difficult owing to enlarged and adherent lymph nodes in the lobar hilum. These nodes were left in situ. On division of the fistula the lumen was seen to be 3 or $4 \mathrm{~mm}$. in diameter. A right lower lobectomy was performed and the fistula in the oesophageal wall closed with a few interrupted sutures of No. 90 linen thread. The post-operative course was uneventful, and the patient was discharged home after three weeks. He has remained well and symptom-free. Histological examination of the resected lobe did not reveal any evidence of tuberculous infection.

\section{Discussion}

The diagnosis of broncho-oesophageal fistula is difficult, unless swallowed barium is seen to enter the fistula and bronchial tree. Symptoms may be slight and due mainly to secondary bronchiectasis or abscess formation.

Clinically the diagnosis can only be made with reasonable certainty if cough is repeatedly precipitated by the passage of food down the oesophagus, and if the expectoration contains particles of undigested food. Sometimes this is more evident in certain postures, particularly when the fistula is dependent. Care has to be taken, however, to differentiate this from the coughing that sometimes occurs after meals in cases of bronchiectasis. Inhalation of food is not always a calamity, and the general condition of the patient may remain good for many years. This observation was made by Levine (1953), who described a man aged 42 with a productive cough for 20 years before the diagnosis was made: his general condition remained good throughout and the fingers did not become clubbed. Coleman and Bunch (1950) state that the four cases they describe (aged 18, 24, 46, and 65 years respectively) were under observation for eight months to 10 years before the correct diagnosis was made.

Maier (1951) describes a man aged 36 and a woman aged 21 who presented with haemoptysis.
Melamed and Zimmerman (1946) describe a similar mode of onset in a woman of 40 . Re- 흠 current pneumonitis, regurgitation of food, retro- $\frac{\bar{\omega}}{\sigma}$ sternal pain, dysphagia, and rales on auscultation $\stackrel{\mathbb{Q}}{\varrho}$ of the chest after drinking water (Ono's sign) have been described in isolated cases.

Coleman and Bunch (1950) analysed 44 cases from the literature where the site of the fistula $\vec{\omega}$ opening was stated, but in only 22 was the opening situated in either stem bronchus or its branches, $\vec{x}$ the remainder being in the larynx or trachea. In four of these the left stem bronchus was involved and in two one of the secondary branches, while the right stem bronchus was involved in 12 cases and one of the secondary branches in four. $\frac{3}{2}$ Another 10 cases from the literature were studied and the proportion of right to left was 6 to 4 . Although the fistulous opening could perhaps be expected to be mainly on the left side, this is not supported by analysis of the reported cases.

PATHOGEnEsis. - The pathogenesis of non-malig- $\vec{\theta}$ nant acquired broncho-oesophageal fistulae is $\mathrm{G}$ varied, inhalation of a foreign body or corrosive being the most common aetiological factor. Disease of the oesophageal wall leading to perforation has been described in syphilis (Bucher and Ono, 1934) and actinomycosis (Vinson and $\mathbb{Q}$ Sutherland, 1926). An aneurysm of the descending aorta, a pyogenic mediastinal abscess from $\frac{3}{3}$ trauma or pyaemia, and a tuberculous abscess $\vec{J}$ from the spine may lead to such a degree of perioesophagitis and bronchial erosion that a fistula results. A diverticulum, either congenital or $\overrightarrow{0}$ acquired by contraction of connective tissue from around an adjacent tuberculous gland, may itself $\underset{-}{*}$ become infected and necrosed. In most of the 3 other cases described a calcified gland has ruptured into both bronchus and oesophagus. The pathogenesis in our case may be perforation of 0 an acquired diverticulum, but the oesophageal mucosal pattern as seen on the radiographs sug-o gested invagination. We believe that an abscess formed in the collapsed right lower lobe and per- -0 forated into the oesophagus. Another possibility $N$ is that a tuberculous gland perforated into both bronchus and oesophagus as the result of a slow process of ulceration on the outer wall of these structures from contact (Kelly and Henderson, 1939) or caseation (Heddaeus, 1889).

TreatMent.-The treatment of these cases is 0 surgical, and it is surprising that of the 75 patients quoted by Coleman and Bunch (1950) only 22ᄋ came to operation, 15 of whom were cured. The outcome in 26 of the 53 untreated patients was 
known ; 16 died in hospital, six remained unaltered, and in four cases the fistula healed spontaneously. When the opening is small, endoscopic cauterization may be of value. Clerf (1933) used $30 \%$ silver nitrate in one case with success, and Clerf, Cooley, and O'Keefe (1943) report two cases where oesophagoscopic application of sodium hydroxide crystals was successful. Melamed and Zimmerman (1946) state that the cauterization method holds promise of cure in oesophagobronchial fistulae.

Direct surgical closure of the fistula has now replaced the endoscopic cautery method, and, when bronchiectasis or secondary abscess has developed, the affected segment or lobe should always be removed.

\section{SUMMARY}

A case of acquired non-malignant bronchooesophageal fistula associated with tuberculous hilar glands in a boy of 12 has been presented. The relevant literature is briefly reviewed.
We are indebted to Dr. Mary Sharp, Dr. K. Mendl, and Dr. T. W. Davies for their co-operation.

\section{REFERENCES}

Bucher, C. J., and Ono, J. (1934). Amer. J. Path., 10, 391.

Clerf, L. H. (1933). Ann. Otol., St. Louis, 42, 920.

- Cooley, E. E., and O'Keefe, J. J. (1943). Surg. Gynec. Obstet., 77,615 .

Coleman, P. F., and Bunch, G. H. (1950). J. thorac. Surg., 19, 542. Heddaeus, J. (1889). Berl. klin. Wschr., 26, 800.

Kelly, A. B., and Henderson, F. L. (1939). J. Laryng., 54, 95.

Levine, I. (1953). J. Amer. med. Ass., 151, 995.

Maier, H. C. (1951). Amer. Rev. Tuberc., 63, 220.

Melamed, A., and Zimmerman, B. M. (1946). Amer. J. Roentgenol., $55,20$.

Monserrat, J. L. (1941). J. Amer. med. Ass., 151, 995.

Morton, D. R., Osborne, J. F., and Klassen, K. P. (1950). J. thorac. Surg., 19, 811.

Nuboer, J. F. (1953). Arch. chir. neerl., 5, 271.

Vinson, P. P., and Sutherland, C. G. (1926). Radiology, 6, 63.

This case was mentioned in the Addendum of an article on "Primary Tuberculosis of the Lung" by Thomas, J. H., Morgan, D. B., and Davies, T. W. (1954). Brit. med. J., 2, 1325. 